\title{
Models for Assessing the Impact of Family Planning on Fertility
}

\author{
Roshan Aryal', Tika Ram Aryal ${ }^{2}$ and M.K. Bhusal ${ }^{2}$ \\ ${ }^{1}$ Maharajgunj Medicine Campus, Kathmandu, Nepal \\ ${ }^{2}$ Central Department of Statistics \\ Tribhuvan University, Kathmandu, Nepal \\ E-mail:traryal@gmail.com
}

\begin{abstract}
This paper tries to assess the impact of family planning (FP) on fertility in Nepal. Nepal Demographic and Health Survey data 2011 were used. Impact of sterilization was found to be highest (about 33 per cent births averted) followed by the combined effect of injectables, pills, condoms and all other traditional methods. The combined impact of sterilization and injectables was found to be 0.60 births per woman. The expected numbers of future children would be 3.09 (excluding sterilization), 3.27 (excluding sterilization or injectables), 3.36 (excluding sterilization or injectables or pills), 3.51 (excluding sterilization or injectables or pills or condoms) and 3.97 (non-user) for zero parity women. The combined impact of sterilization and injectables would be 0.60 births per woman. Findings may help researchers and policy-makers for designing effective FP policy of a country.
\end{abstract}

Keywords:- Sterilization, period parity progression ratio, reproduction, contraceptive methods, birth order.

\section{INTRODUCTION}

Total fertility rate (TFR) is a reliable measure, which influenced by a set of intermediate variables (Davis \& Blake, 1956). Bongaarts and Potter (1983) revised intermediate components with Proximate Determinants of Fertility, using data from 41 developed and developing countries where about 96 percent of total variation in fertility due to marriage, contraception, lactational infecundability and induced abortion. The reproduction is normally taking place during teens of life; however, there is a big variation in childbearing performance in the society (Aryal 2012, Riley et al. 1993). Fertility behavior is changing over time and numerous studies have been conducted elsewhere (Diamond \& Rutenberg, 1995; Vander Post, 1992). Among population of high fertility and low contraceptive practices, the unwanted fertility was reported to be higher (Aryal 2005, Kulkarni \& Choe 1998, Riley et al. 1993) where the extent of unwanted fertility is likely to vary from society to society.

Period parity progression ratios (PPPR) methods reflect the tempo of cohort fertility, which gives an additional advantage to look at trends in TFR, which facilitates to make comparison separately regarding progression of a specific parity to higher order parity (Bhrolchain, 1987, Feeney \& Yu 1994, Luther et al. 1990, Rao \& Balkrishnan 1989). The knowledge of FP is almost universal and about 99 percent of currently married women heard of at least one method of FP (Aryal 2011). Contraceptive prevalence rate- the percentage currently using contraception among couples with the women of reproductive age (15-49) - has been reported at 49.7 percent in 2011. The percentage of contraceptive use is increased rapidly in Nepal during the recent past whereas the fertility level declines from 6.33 in 1976 to 4.60 in 1996 to 4.1 in 2001 , to 3.1 in 2001 and to 2.6 births per woman in 2011 (MOPH 2011). However, the departure in current fertility from the potential fertility may be taken as the overall impact of acceptance of different methods of FP on fertility provided that the nuptuality pattern had been constant and natural fertility did not change otherwise. If the fertility is computed after excluding the women who are not exposed to conception due to use of contraception, it may be taken as the natural fertility (Aryal 2011, Mosher \& Jones 2010).

The extent of reduction in fertility due to the use of various contraceptive methods viz. sterilization alone, sterilization or injectables, sterilization or injectables or pills, sterilization or injectables or pills or condoms used has been assessed separately to know the amount of averted births due to FP (Kost et al. 2008). Overall impact of FP programs depends upon the number of users of different methods. Effectiveness varies widely and sterilization has been found most effective method followed by injectables, pills and then conventional contraceptives (CCs) (Aryal 2005, Pathak 1998, Ram \& Pathak 1993, U N 1997).

Nepal is one of the moderately high fertility experienced developing country in 1990s. Its fertility transition is of particular interest in three ways (Caldwell, 1998) i.e. "(i) the low per capita income at which it is occurring, (ii) 
the different topography of the country, which divides the population into those with easy access to the outside world who have joined the global economy and exhibit declining fertility and those without roads or school who are still characterized by stable high fertility, and (iii) the reliance for most fertility control on sterilization". In recent times, it has a declining trend in fertility in spite of limited resources of basic necessities for the people (Aryal 2002, MOPH 2011).

Evidence suggests that Nepal has also been experiencing a gradual decline in fertility over the last several years in spite of a limited resources made for the improvement of basic necessities of the people, viz. literacy, urbanization, industrialization, status of female, female's age at marriage, infant mortality, maternal mortality, level of income. However, there is still a high fertility experienced population in a country. In this context, this paper tries to discuss the procedure to assess the FP impact on fertility by using Nepal Demographic and Health Survey data 2011. The approach based on parity progression ratio has been applied to assess the extent of reduction in fertility due to the use of various FP methods.

\section{MATERIALS AND METHODS}

\section{Procedure to assess the impact of FP on fertility}

The procedures applied here was based on PPPR in absence of sterilization may be obtained by excluding those women who are already sterilized. Let us call this group as regime II of the fertility regulation. In the absence of sterilization or injectables, PPPR may be obtained by excluding those women who are either sterilized or used injectables. We name this group of women as regime III. Similarly, in the absence of sterilization or injectables or pills and sterilization or injectables or pills or condoms, PPPR may be obtained. The groups of women in this category are denoted as regimes IV and V respectively. Regime VI denotes the groups of women with the level of fertility in the absence of all contraception methods. Clearly, regime I represents the level of fertility in presence of contraception. PPPR, thus obtained, is used to calculate the value of TFR, and probability of ever having a specific order of birth or probability of ever becoming a mother of $i$ children $\left(F_{i}\right)$ and probability of having the final parity $\mathrm{i}\left(\mathrm{B}_{\mathrm{i}}\right)$ i.e. parity distribution.

Previous studies have attempted to discuss distributions of fertility through changes in the contemporary parity distributions of women in the population (Pandey et al. 1997, Pandey \& Suchindran 1995). Here, parity distribution is obtained to see the pattern of birth averted from the use of FP methods. Since $P_{i},(i \geq 0)$ is the probability that a woman of parity $i$ is proceeding for the next higher parity $(\mathrm{i}+1)$, the probability of ever bearing $i$ children $\left(F_{i}\right)$ over the reproductive age range (15-49) is obtained as:

$$
\begin{aligned}
& F_{i+1}=\coprod_{i=0}^{k} P_{i} \\
& \text { and } \mathrm{F}_{0}=1
\end{aligned}
$$

Further, if $\mathrm{B}_{\mathrm{i}}$ denotes the probability that a woman finally have $i$ parity $(i \geq 0)$ in her reproductive span (15-49 years), then

$$
B_{i}=F_{i}-F_{i+1}
$$

The expected number of births of order i, per woman, is simply given by:

$$
\frac{\sum_{i=0}^{n} F_{i}}{F_{0}}
$$

and the expected number of future children for a woman currently of parity $i$ is given as:

$$
\frac{\left.F_{i+1}+F_{i+2}+F_{i+3}+. .\right)}{F_{i}}
$$

The Nepal Demographic and Health Survey 2011 data are extracted to apply the discussed indirect techniques. The data are analyzed in order to see the impact of FP methods on fertility taking 2010-11. PPPRs are used to study the impact of a FP method on fertility under different regimes of contraception. The following regimes of contraception will be defined by taking into account of the practices of different contraceptive methods. Thus regimes of fertility regulation programs due to use of different FP methods are:

I- The prevailing fertility levels due to use of all FP methods for period of 2010-11.

II- Excluding women those who had sterilized (male/female) during the period of 2010-11.

III- Excluding women those who had sterilized (male/female) or used injectables during the period of 2010-11.

IV- Excluding women those who had sterilized (male or female) or used injectables or used pills during the period of 2010-11.

V- Excluding women those who had sterilized (male/female) or used injectables or used pills or used condoms during the period of 2010-11.

VI- Excluding women those who had sterilized (male/female) or used injectables or used pills or used condoms or all other FP methods including traditional methods imply that non-users' of FP methods during the period of 2010-11. 


\section{RESULTS}

The levels of fertility based on PPPRs vary according to the regimes of fertility regulation. Table 1 explains the PPPRs of the regimes of fertility regulations of the period 2010-11. For instance, for regime I, the prevailing levels of FP method, given in the last column showing the values of PPPR in the presence of contraception indicated that the second and higher order PPPR decreased with the increased parity order. Similarly, regime II indicated the PPPR by excluding mothers who had sterilized according to parity order of woman for the year 201011. Regime III showed the PPPR by excluding both women those who sterilized as well as used injectables. Regime IV displayed the PPPRs for woman who were sterilized or used injectables or Pills or condom. Regime $\mathrm{V}$ displayed the PPPRs for woman who were sterilized or used injectables or Pills or condom or all other traditional methods. Regime VI presents the fertility level for women those who had not used any method of contraception.

Table 1. PPPRs under different regimes of fertility regulations (2010-11)

\begin{tabular}{|c|c|c|c|c|c|c|}
\hline \multirow{2}{*}{$\begin{array}{c}\text { Parity } \\
(\mathbf{i})\end{array}$} & \multicolumn{7}{|c|}{ Parity progression ratio $\left(\mathbf{P}_{\mathbf{i}}\right)$} \\
\hline & VI & V & IV & III & II & I \\
\hline 0 & 0.9879 & 0.9898 & 0.9873 & 0.9887 & 0.9897 & 0.9869 \\
\hline 1 & 0.9859 & 0.9710 & 0.9705 & 0.9598 & 0.8898 & 0.7930 \\
\hline 2 & 0.8551 & 0.7323 & 0.7345 & 0.6839 & 0.6720 & 0.4113 \\
\hline 3 & 0.5972 & 0.4935 & 0.3097 & 0.2816 & 0.2500 & 0.2903 \\
\hline 4 & 0.3039 & 0.1516 & 0.1416 & 0.1379 & 0.1129 & 0.1425 \\
\hline 5 & 0.2686 & 0.0806 & 0.0767 & 0.0747 & 0.0511 & 0.0726 \\
\hline 6 & 0.1307 & 0.0548 & 0.0501 & 0.0489 & 0.0376 & 0.0511 \\
\hline 7 & 0.0318 & 0.0226 & 0.0147 & 0.0201 & 0.0215 & 0.0162 \\
\hline 8 & 0.0212 & 0.0194 & 0.0118 & 0.0172 & 0.0188 & 0.0084 \\
\hline 9 & 0.0177 & 0.0129 & 0.0088 & 0.0144 & 0.0161 & 0.0036 \\
\hline
\end{tabular}

Fig. 1 clearly shows that PPPRs were dropped from marriage to first birth order and after the first birth order, the dropping pattern in proceeding births to next parity was found to be faster with fastest among the fertility regimes II to V. The areas showing between lines represent as the impact of FP methods on fertility due to the respective regimes of fertility.

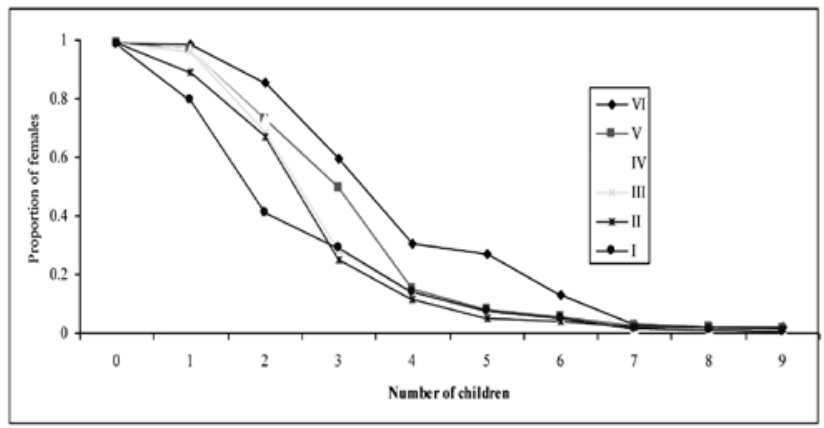

Fig. 1. Proportion of females by No. of children (2010-11)
Table 2. Probability of ever having a mother of $i$ children under the regimes of fertility regulations (2010-11)

\begin{tabular}{|c|c|c|c|c|c|c|}
\hline \multirow{2}{*}{$\begin{array}{c}\text { Parity } \\
\text { (i) }\end{array}$} & \multicolumn{5}{|c|}{ Probability of ever having i children $\left(\mathbf{F}_{\mathbf{j}}\right)$} \\
\hline & VI & V & IV & III & II & I \\
\hline 0 & 1.0000 & 1.0000 & 1.0000 & 1.0000 & 1.0000 & 1.0000 \\
\hline 1 & 0.9879 & 0.9898 & 0.9873 & 0.9887 & 0.9897 & 0.9869 \\
\hline 2 & 0.9739 & 0.9611 & 0.9582 & 0.9489 & 0.8806 & 0.7826 \\
\hline 3 & 0.8328 & 0.7037 & 0.7038 & 0.6490 & 0.5918 & 0.3219 \\
\hline 4 & 0.4973 & 0.3473 & 0.2180 & 0.1828 & 0.1480 & 0.0935 \\
\hline 5 & 0.1511 & 0.0527 & 0.0309 & 0.0252 & 0.0167 & 0.0133 \\
\hline 6 & 0.0406 & 0.0042 & 0.0024 & 0.0019 & 0.0009 & 0.0010 \\
\hline 7 & 0.0053 & 0.0002 & 0.0001 & 0.0001 & 0.0000 & 0.0000 \\
\hline 8 & 0.0002 & 0.0000 & 0.0000 & 0.0000 & 0.0000 & 0.0000 \\
\hline
\end{tabular}

Probability of ever having a mother of $i^{\text {th }}$ parity under different regimes of fertility regulations (I to VI) can be obtained by using PPPRs. Table 2 displays the probability of ever bearing $i$ children according to the parity order for the regimes of I to VI fertility regulations during 201011. The probability for proceeding marriage to first order birth was found to be more or less similar in nature and the probability of first to second and second to third order births was steadily decreasing as faster for the regimes I and followed by regimes II, III, IV, V and VI, which indicating for controlling or limiting the number of births per women for higher order parity during 2010-11.

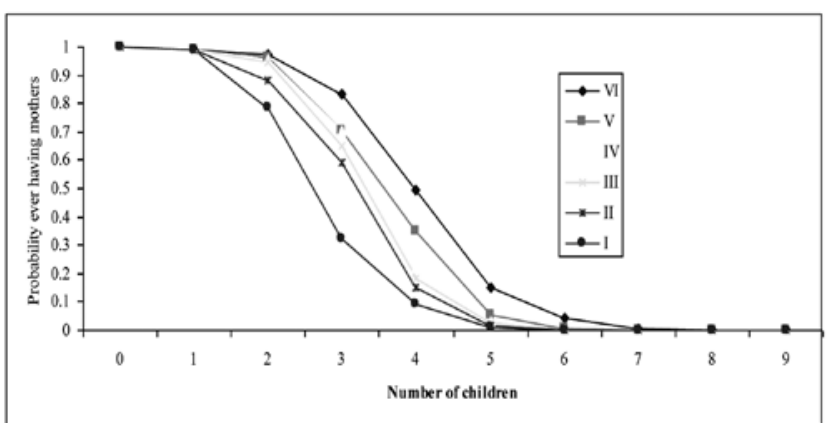

Fig. 2. Probability of ever having a mother i children under different regimes of family planning methods (2010-11)

Fig. 2 clearly shows that the probability ever having mothers' declines steadily after second and third births for all the regimes of fertility regulation programs and thereafter it declines rapidly with increased parity order. A faster declining tempo for regime I followed by the regimes II, III, IV, V and VI respectively can also be found during 2010-11. It also shows the proportion of women according to the number of children for the fertility regimes and clearly presents the FP impact on fertility. Results also shows the fertility regulation due to the use of FP methods where the lines between the regime I to regime VI, and the area showing between lines was accounted as the impact of FP methods during 
2010-11. It was also shown that sterilization was found higher impact of FP on fertility than other methods.

Table 3. Birth distribution under different regimes of fertility regulations (2010-11)

\begin{tabular}{|c|c|c|c|c|c|c|}
\hline \multirow{2}{*}{$\begin{array}{c}\text { Parity } \\
\text { (i) }\end{array}$} & \multicolumn{6}{|c|}{ Birth order distribution (B) } \\
\cline { 2 - 7 } & VI & V & IV & III & II & I \\
\hline 0 & 0.0121 & 0.0102 & 0.0127 & 0.0113 & 0.0103 & 0.0131 \\
\hline 1 & 0.0140 & 0.0287 & 0.0291 & 0.0398 & 0.1091 & 0.2043 \\
\hline 2 & 0.1411 & 0.2573 & 0.2544 & 0.2999 & 0.2888 & 0.4607 \\
\hline 3 & 0.3355 & 0.3564 & 0.4858 & 0.4662 & 0.4439 & 0.2284 \\
\hline 4 & 0.3462 & 0.2947 & 0.1871 & 0.1575 & 0.1312 & 0.0801 \\
\hline 5 & 0.1105 & 0.0484 & 0.0285 & 0.0233 & 0.0159 & 0.0123 \\
\hline 6 & 0.0353 & 0.0040 & 0.0022 & 0.0018 & 0.0008 & 0.0009 \\
\hline 7 & 0.0051 & 0.0002 & 0.0001 & 0.0001 & 0.0000 & 0.0000 \\
\hline 8 & 0.0002 & 0.0001 & 0.0000 & 0.0000 & 0.0000 & 0.0000 \\
\hline 9 & 0.0001 & 0.0000 & 0.0000 & 0.0000 & 0.0000 & 0.0000 \\
\hline
\end{tabular}

Table 3 portrays the birth order distributions by parity for the different fertility regimes. An analysis of the distribution of birth order implies the fertility levels for different regimes of fertility regulations programs and that provides a clear picture of FP impact on fertility. For instance, the level of fertility based on PPPRs varies according to the regimes of fertility regulation and thereby the birth distributions. The proportion of childlessness was found to be 1.03 per cent for women who used contraception, whereas it was 1.21 per cent for women who did not use any method. The probability for proceeding third parity was found to be .3462 among non-users' whereas it was .2284 for prevailing fertility regimes.

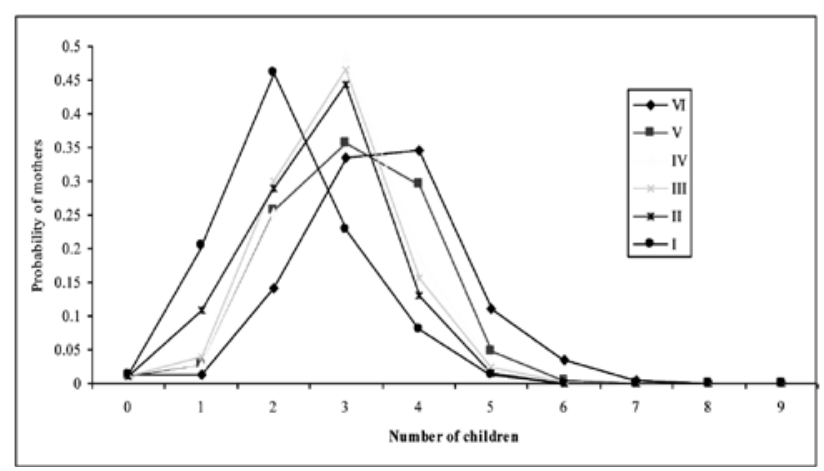

Fig. 3. Birth distribution under different regimes of FP methods (2010-11)

The birth distribution shows the highest peaks in the proceeding from second, third and fourth order of births during 2010-11 respectively to the regimes of I, II, III, IV, $\mathrm{V}$ and VI. Fig. 3 clearly indicates the probability of birth distributions and also shows the chance of childlessness with respect to the different fertility regimes, which finally shows the impact of FP methods during 2010-11. The shape of the curve also signifies the probability of the impact of FP methods in accordance with the different regimes of fertility regulation programs.

The TFRs above parity i (for $i=1,2, \ldots, 9$ ) under different fertility regimes are presented in Table 4 . It was found that fertility levels were found to be $2.20,2.63,2.80,2.90$, 3.06 and 3.49 births per woman for the regimes I, II, III, IV, V and VI respectively for the period 2010-11. Regime VI naturally presents the fertility level for women who had not used any method of FP. For this regime, the TFR was found to be 3.49 births per woman for the period 2010-11. An overall impact of FP can be observed that about 1.29 births per woman for the period 2010-11 have been averted due to FP methods. Impact of sterilization was found to be highest (about 33 percent births averted) followed by the combined effect of injectables, pills, condoms and all other traditional methods. Fig. 4 shows the period TFR above the parity i under different fertility regimes, which indicating the impact of FP methods on fertility during 2010-11.

Table 4. TFR above parity i for the different regimes of fertility regulations (2010-11)

\begin{tabular}{|c|c|c|c|c|c|c|}
\hline \multirow{2}{*}{ Parity(i) } & \multicolumn{7}{|c|}{ TFR above parity i } \\
\cline { 2 - 7 } & VI & V & IV & III & II & I \\
\hline 0 & 3.49 & 3.06 & 2.90 & 2.80 & 2.63 & 2.20 \\
\hline 1 & 2.50 & 2.07 & 1.91 & 1.81 & 1.64 & 1.21 \\
\hline 2 & 1.53 & 1.11 & 0.96 & 0.86 & 0.76 & 0.43 \\
\hline 3 & 0.69 & 0.40 & 0.25 & 0.21 & 0.17 & 0.11 \\
\hline 4 & 0.20 & 0.06 & 0.03 & 0.03 & 0.02 & 0.01 \\
\hline 5 & 0.05 & 0.00 & 0.00 & 0.00 & 0.00 & 0.00 \\
\hline 6 & 0.01 & 0.00 & 0.00 & 0.00 & 0.00 & 0.00 \\
\hline 7 & 0.00 & 0.00 & 0.00 & 0.00 & 0.00 & 0.00 \\
\hline 8 & 0.00 & 0.00 & 0.00 & 0.00 & 0.00 & 0.00 \\
\hline 9 & 0.00 & 0.00 & 0.00 & 0.00 & 0.00 & 0.00 \\
\hline
\end{tabular}

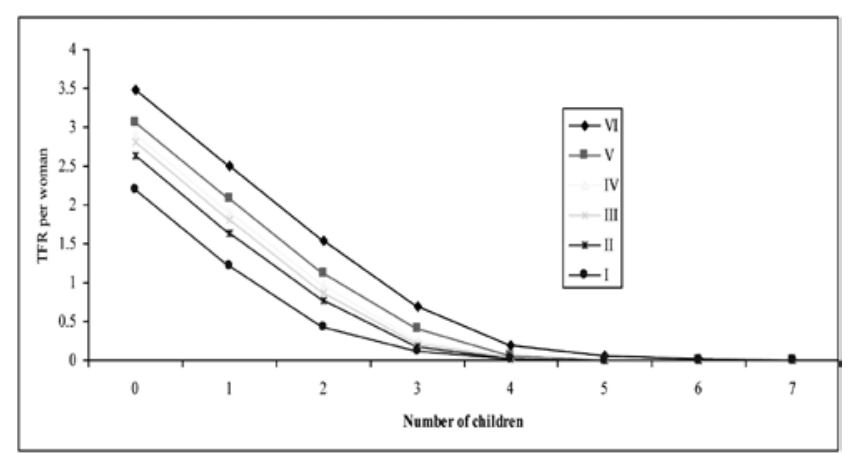

Fig. 4. TFR above parity $i$ under different fertility regimes of FP methods (2010-11)

Table 5 explains the expected number of future children for a woman currently of parity $i(i=1,2, \ldots 9)$ under the different regimes of fertility regulations. The expected numbers of future children would be 2.63 (excluding sterilization), 2.80 (excluding sterilization or injectables), 
2.90 (excluding sterilization or injectables or pills), 3.06 (excluding sterilization or injectables or pills or condoms) and 3.49 (non-user) for zero parity women whereas among women of first parity, the expected number of future children would be 1.21, 1.64, 1.81, $1.91,2.07$ and 2.50 births per woman. Similarly expected number of future children for the second parity would be $.55, .86, .91,1.0,1.15$ and 1.57 births per woman under the different fertility regimes of I, II, III, IV, V and VI respectively. Fig. 5 shows the FP impact on fertility for the future number of children under the different fertility regimes if prevailing situation will be continued in future. An impact of sterilization would be 0.43 births per woman for the period of 2010-11 and the amount of averted births would be $0.18,0.11,0.15$ and 0.46 births per woman due to use of injectables, pills, condoms and all other traditional methods including implant, IUD, period withdrawal, etc. respectively. The combined impact of sterilization and injectables would be 0.60 births per woman for the period of 2010-11.

Table 5. Expected No. of children above parity i for the different regimes of fertility regulations (2010-11)

\begin{tabular}{|c|c|c|c|c|c|c|}
\hline \multirow{2}{*}{$\begin{array}{c}\text { Parity } \\
\text { (i) }\end{array}$} & \multicolumn{7}{|c|}{ Expected future children } \\
\cline { 2 - 7 } & VI & V & IV & III & II & I \\
\hline 0 & 3.49 & 3.06 & 2.90 & 2.80 & 2.63 & 2.20 \\
\hline 1 & 2.53 & 2.09 & 1.94 & 1.83 & 1.66 & 1.23 \\
\hline 2 & 1.57 & 1.15 & 1.00 & 0.91 & 0.86 & 0.55 \\
\hline 3 & 0.83 & 0.57 & 0.36 & 0.32 & 0.28 & 0.33 \\
\hline 4 & 0.40 & 0.16 & 0.15 & 0.15 & 0.12 & 0.15 \\
\hline 5 & 0.30 & 0.09 & 0.08 & 0.08 & 0.05 & 0.08 \\
\hline 6 & 0.13 & 0.06 & 0.05 & 0.05 & 0.04 & 0.05 \\
\hline 7 & 0.03 & 0.02 & 0.01 & 0.02 & 0.02 & 0.02 \\
\hline 8 & 0.02 & 0.02 & 0.01 & 0.02 & 0.02 & 0.02 \\
\hline 9 & 0.00 & 0.00 & 0.00 & 0.00 & 0.00 & 0.00 \\
\hline
\end{tabular}

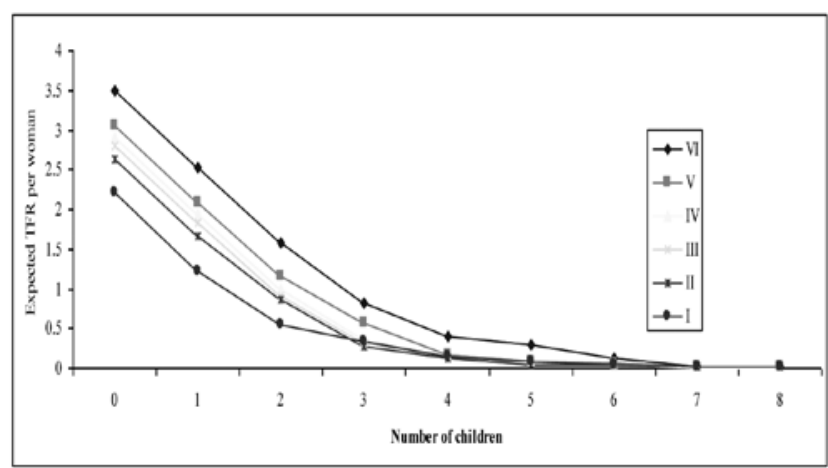

Fig. 5. Expected No. of children above parity i under different fertility regimes of FP methods (2010-11)

\section{CONCLUSION}

This study presents the procedures to assess FP impact on fertility through PPPRs. Impact of sterilization was found to be highest (about 33 per cent births averted) followed by the combined effect of injectables, pills, condoms and all other traditional methods. An impact of sterilization was found to be 0.43 births per woman and the amount of averted births was $0.18,0.11,0.15$ and 0.46 births per woman due to use of injectables, pills, condoms and all other traditional methods including implant, IUD, period withdrawal, etc. respectively. The combined impact of sterilization and injectables was found to be 0.60 births per woman. The expected numbers of future children would be 3.09 (excluding sterilization), 3.27 (excluding sterilization or injectables), 3.36 (excluding sterilization or injectables or pills), 3.51 (excluding sterilization or injectables or pills or condoms) and 3.97 (non-user) for zero parity women. The expected number of future children would be 2.12, 2.31, 2.40, 2.56, and 3.01 births per woman. Expected number of future children for the second parity would be $1.20,1.35,1.45,1.58$, and 2.07 births per woman for the first parity under different fertility regimes of I, II, III, IV, V and VI respectively. An impact of sterilization would be 0.42 births per woman and the amount of averted births would be $0.18,0.11$, 0.15 and 0.46 births per woman due to use of injectables, pills, condoms and all other traditional methods including implant, IUD, period withdrawal respectively. The combined impact of sterilization and injectables would be 0.60 births per woman. Low impact of different FP methods on fertility may be due to low effectiveness of contraceptive use or due to traditional socio-economic pillars as well as cultural perspectives of the people of Nepal.

\section{ACKNOWLEDGEMENTS}

T.R. Aryal would like to thank to the University Grants Commission, Nepal for providing financial supports.

\section{REFERENCES}

Aryal, T.R. 2011. Fertility Dynamics of Nepal. Ekta Books, Kathmandu, Nepal.

Aryal, T.R. 2013a. Application of demographic models to investigate levels, differentials and determinants of fertility of Nepal. Journal of Institutes of Science and Technology 18 (1): 148-156.

Aryal, T.R. 2013b. Tempo-distortions in fertility: application of Nepal Demographic Health Survey 2011 Data. Journal of Institutes of Science and Technology 18 (2): 116-120.

Aryal, T.R. 2014. Unwanted fertility differentials of Nepalese mothers. Journal of Institutes of Science and Technology 19 (1): 45-51

Aryal, T.R. 2002. Some Demographic Models and Their Applications with Reference to Nepal. $\mathrm{PhD}$ thesis, Department of Statistics, BHU, India. 
Aryal, T.R. 2005. Impact of family planning programs on fertility: alternative methodological perspectives. The Economic Journal of Nepal $\mathbf{2 8}$ (1): $38-44$.

Aryal, T.R. 2006. The fertility impact of changes in the timing of childbearing in Nepal. Nepal Journal of Science and Technology 7: 47-52.

Bhrolchain, M.N. 1987. Period parity progression ratios and birth intervals in England and Wales, 19411971: a synthetic life table analysis. Population Studies 41: 103-125.

Bongaarts, J. and Potter, R.G. 1983. Fertility, Biology and Behavior: Analysis of the Proximate Determinants of Fertility. Academic Press, New York.

Caldwell, J.C. 1998. The Global fertility transition and Nepal. Contributions to Nepalese Studies 25: 1-7.

Davis, K. and Blake, J. 1956. Social structure and fertility: An analytical framework. Economic Development and Cultural Change 4(2): 117121.

Diamond, I., Rutenberg, N. 1995. Recent trends in fertility in Botswana. Journal of International Development 7(1): 145-161.

Feeney, G. and Yu, J. 1994. Below replacement fertility in China? A close look at recent evidence. Population Studies 48: 381-394.

Kost, K., Singh, S., Vaughan, B., Trussell, J. and Bankole, A. 2008. Estimates of contraceptive failure from the 2002 National Survey of Family Growth. Contraception 77: 10-21.

Kulkarni, S. and Choe, M.K. 1998. Wanted and Unwanted Fertility in Selected States of India. National Family Health Survey Subject Reports, Number 6, East-West Center Program on Population Honolulu, Hawaii, U.S.A.

Luther, N.Y., Feeney ,G. and Zhang, W. 1990. One child families or Baby Boom? Evidence from China's: 1987 One- per- Hundred Survey. Population Studies 44: 341-357.
MOPH. 2011. Nepal Demographic and Health Survey 2011 Preliminary Report. Ministry of Population and Health. Kathmandu, Nepal.

Mosher, W.D., and Jones, J. 2010. Use of contraception in the United States: 1982-2008. Vital Health Statistics Series, 23(29):1-44. Hyattsville, MD: National Center for Health Statistics.

Pandey, A. \& Suchindran, C.M. 1995. Some analytical models to estimate maternal age at birth using age specific fertility rates. Sankhya, Series- $B$ 57(1): $142-150$.

Pandey, A., Saxena, N.C. and Singh, K.K. 1997. Estimation of parity progression ratios from birth order statistics. Genus: A Journal of Population Studies, LIII (1\&2): 177-188.

Pathak, R.S. 1998. Family planning programs effort, contraceptive prevalence and fertility: the case of Nepal in South Asia. Nepal Population Journal 7(6): 45-50.

Ram, F. and Pathak, K.B. 1993. Evaluation of Family Programs in India using Different Approaches: An Assessment. Project Report, International Institute for the study of Population Sciences, Deonar, Bombay.

Rao, K.V. and Balkrishnan, T.R. 1989. Timing of first birth and second birth spacing in Canada. Journal of Bi-social Science 21: 293-300

Riley, A.P., Samuelsion, J.L. and Huffman, S.L. 1993. The relationship of age at menarche and fertility in undernourished adolescents. Biomedical and demographic determinants of reproduction (Ed. R. Grey et al.): 50-84.

U.N. 1997. Family Building and Family Planning Evaluation. Department of Economic and Social Affairs: Population Division, ST/ESA/SER.R/148, New York, U S A.

Vander, P.C. (1992). Regional patterns of fertility transition in Botswana. Geography 77(2): 109-122. 